\title{
Nonunitarizable Representations and Random Forests
}

\author{
Inessa Epstein ${ }^{1,2}$ and Nicolas Monod ${ }^{1}$ \\ ${ }^{1}$ Ecole Polytechnique Fédérale de Lausanne, 1015 Lausanne, Switzerland \\ Correspondence to be sent to: nicolas.monod@epfl.ch
}

We establish a connection between Dixmier's unitarizability problem and the expected degree of random forests on a group. As a consequence, a residually finite group is nonunitarizable if its first $L^{2}$-Betti number is nonzero or if it is finitely generated with nontrivial cost. Our criterion also applies to torsion groups constructed by Osin, thus providing the first examples of nonunitarizable groups without free subgroups.

\section{Introduction}

If an operator $P$ is conjugated to a unitary operator, then it is uniformly bounded in the sense that $\sup _{n \in \mathbf{Z}}\left\|P^{n}\right\|$ is finite. The classical 1947 article by Szőkefalvi-Nagy [49] establishes the converse. A remarkable feature of Szókefalvi-Nagy's short proof is that it uses the Banach-Mazur "generalized limits."

More generally, a representation $\pi$ of a group $G$ on a Hilbert space $V$ is called unitarizable if there is an invertible operator $T$ of $V$ such that $T \pi(g) T^{-1}$ is unitary for all $g \in G$. In that case, $\pi$ is necessarily uniformly bounded in the sense that $\sup _{g \in G}\|\pi(g)\|$ is finite. Both Dixmier [12] and Day [11] noticed that the very proof of Szókefalvi-Nagy establishes that every amenable group is unitarizable, meaning that all its uniformly bounded representations are unitarizable. Indeed, a group is amenable by definition if it admits an invariant mean, i.e. a generalized Banach limit.

${ }^{2}$ Present address: Caltech, Pasadena, CA 91125, USA.

Received December 8, 2008; Revised May 3, 2009; Accepted May 28, 2009

Communicated by Prof. Marc Burger

(C) The Author 2009. Published by Oxford University Press. All rights reserved. For permissions,

please e-mail: journals.permissions@oxfordjournals.org. 
Dixmier asked in [12, Section 5] whether unitarizability characterizes amenability; the present note contributes to this question. For more background, we refer to [43].

A property very much opposed to amenability is the nonvanishing of the first $L^{2}$-Betti number $\beta^{1}$ (to be briefly recalled below; for a detailed discussion, see $[15,37])$.

Theorem 1.1. Let $G$ be a residually finite group. If $\beta^{1}(G)>0$, then $G$ is not unitarizable.

A similar property is that the cost $\mathscr{C}$ studied in [18] be larger than one. As suggested by Abért, the arguments leading to the previous result have a parallel with cost.

Theorem 1.2. Let $G$ be a finitely generated residually finite group. If $\mathscr{C}(G)>1$, then $G$ is not unitarizable.

In fact, Dixmier first asked whether any group at all fails to be unitarizable; this was answered in 1955 when Ehrenpreis-Mautner [16] showed that the complementary series of $\mathrm{SL}_{2}(\mathbf{R})$ can be extended to uniformly bounded representations that are not unitarizable. A detailed treatment was given by Kunze-Stein [28].

By general properties of unitarizability, the existence of any nonunitarizable group implies that the free group $F_{2}$ is nonunitarizable (see [43]). Very explicit nonunitarizable representations of $F_{2}$ were constructed in the 1980s [8, 39, 45]. It follows by induction of representations that any group containing $F_{2}$ as a subgroup is nonunitarizable.

Until now, there was no example of a nonunitarizable group not containing $F_{2}$. In fact, even the existence of nonamenable groups without $F_{2}$ as a subgroup was a long-standing open problem in group theory, not solved until the 1980s $[1,40]$.

We aim to construct nonunitarizable representations under weaker assumptions than the existence of a free subgroup. A result of Gaboriau-Lyons [22], notably using [23] and [46], provides an $F_{2}$-action on the Bernoulli percolation of any nonamenable countable group $G$ in such a way that $F_{2}$ can be thought of as a "random subgroup" of $G$, even when $G$ has no actual such subgroup. It was suggested in [38] (Problem N) to apply an induction procedure for specific representations of random subgroups in order to answer Dixmier's question. In fact, the first use of [22] toward a cohomological 
question asked in [24, Section 10] can be found in [38, Section 5] and the second use is the ergodic-theoretical result [17].

We shall follow the above strategy, but using the language of random forests instead of relying on [22]. A forest on a group $G$ is a subset $F \subseteq G \times G$ such that the resulting graph $(G, F)$ has no cycles. The collection $\mathscr{F}_{G}$ of all forests on $G$ is a closed $G$-invariant subspace of the compact $G$-space of all subsets of $G \times G$ if we consider the usual product topology (i.e. pointwise convergence) and the left diagonal $G$-action. A random forest is a $G$-invariant Borel probability measure on $\mathscr{F}_{G}$. By $G$-invariance, the expected degree of a vertex in a random forest does not depend on the vertex; we call it the expected degree $\operatorname{deg}(\mu)$ of the random forest $\mu$. Similarly, we define the width width $(\mu)$ as the number of vertices that neighbor a given vertex with positive probability. We shall be interested in forests with finite width. Of course, one has $\operatorname{deg}(\mu) \leq \operatorname{width}(\mu)$.

Theorem 1.3. Let $G$ be a unitarizable group. Then the quantity

$$
\frac{\operatorname{deg}(\mu)^{2}}{\operatorname{width}(\mu)}
$$

is bounded uniformly over all random forests $\mu$ (of finite width) defined on all countable subgroups of $G$.

Remark 1.4. We only made the countability assumption in order to have a metrizable space of forests on which the probability is defined. This is an inessential restriction; in any case, unitarizability is a countably determined property [44,0.10]. Notice also that all trees in a forest of finite width are countable.

Using known estimates on specific random forests, Theorem 1.3 implies the following statement, wherein the $\operatorname{rank} \operatorname{rk}(H)$ denotes the minimal number of generators of a group $H$.

Theorem 1.5. Let $G$ be a unitarizable group. Then the quantities

$$
\frac{\left(\beta^{1}(H)\right)^{2}}{\operatorname{rk}(H)}, \quad \frac{(\mathscr{C}(H))^{2}}{\operatorname{rk}(H)}
$$

are bounded uniformly over all finitely generated subgroups $H$ of $G$.

We asked Osin whether one knows examples of groups without nonabelian free subgroup and violating the above bound involving $\beta^{1}$. It turns out that Osin can construct torsion groups with this property (using among others [47]); for this and more, we refer 
to the forthcoming [41]. Thus, Osin's examples allow us to deduce the following from Theorem 1.5.

Corollary 1.6. There exist nonunitarizable torsion groups.

We shall begin by proving Theorem 1.3 in Section 2 . This result makes it desirable to investigate general constructions of forests with large expected degree. Indeed, Theorems 1.1 and 1.2 will be deduced by considering specific models of random forests and using known estimates for their degrees. In Section 3, we include an expository account of the required properties of the free uniform spanning forest and reduce Theorem 1.1 to Theorem 1.3. The reduction of Theorem 1.2 to Theorem 1.3 in Section 4 follows similar lines but using the minimal spanning forest. Strictly speaking, one could reduce Theorem 1.1 to Theorem 1.2 except for the finite generation issue discussed in Section 5; we prefer to present a more detailed account of the relation between $L^{2}$-Betti numbers and forests and be more concise in Section 4.

Section 5 discusses the context and further directions of research; we point out for instance that any nonamenable finitely generated group admits a random forest with nontrivial (i.e. $>2$ ) expected degree.

\section{Forests and Littlewood}

— ¿Usted sin duda querrá ver el jardín? [...]

- ¿El jardin?

-El jardin de los senderos que se bifurcan.

(J. L. Borges, El jardín de senderos que se bifurcan (The Garden of Forking Paths), 1941.)

We follow Serre's conventions [48] for graphs, which are thus pairs $(V, E)$ of vertex and edge sets with structural maps $E \rightarrow V, e \mapsto e_{ \pm}$and $E \rightarrow E, e \mapsto \bar{e}$. Recall that the underlying "geometric" edges consist of sets of opposed edges $e, \bar{e}$. In the case of simple graphs, i.e. without loops or multiple geometric edges (such as forests), one shall always consider $E$ as a subset of $V \times V$ invariant under the canonical involution and not meeting the diagonal. Recall also that an orientation is a fundamental domain for the involution in $E$.

Given a group $G$, we define the space $\mathscr{G}_{G}$ of all (simple) graphs on $G$ as the subset $\mathscr{G}_{G} \subseteq 2^{G \times G}$ of all subsets $E \subseteq G \times G$ defining a simple graph $(G, E)$. The space $2^{G \times G}$ is compact for the product topology and has a natural $G$-action by left multiplication; 
since $\mathscr{G}_{G}$ is closed and invariant, it is itself a compact $G$-space. A random graphing of $G$ is a $G$-invariant probability measure on $\mathscr{G}_{G}$.

We now consider the closed $G$-invariant subspace $\mathscr{F}_{G} \subseteq \mathscr{G}_{G}$ of forests and recall from the Introduction that a random forest is a random graphing supported on $\mathscr{F}_{G}$. We shall not be interested in the forest of width zero. We denote by $\mathscr{F}_{G}^{+}$the set of all orientations of all forests and view it as a closed $G$-invariant subspace of the compact $G$-space of subsets of $G \times G$. There is a canonical $G$-equivariant quotient map $\mathscr{F}_{G}^{+} \rightarrow \mathscr{F}_{G}$.

Example 2.1. Suppose that $S \subseteq G$ is a subset freely generating a free subgroup. Then we obtain a forest $F \in \mathscr{F}_{G}$ by $F=\left\{\left(g, g^{\prime}\right): g^{-1} g^{\prime} \in S \cup S^{-1}\right\}$. This forest is $G$-fixed and hence is a (deterministic) random forest.

Example 2.2. Suppose that $G$ is finite and already endowed with a graph structure $(G, E)$. The uniform measure on the set of all spanning trees of $(G, E)$ is a random forest. Aside from the notion of $G$-invariance, this random forest makes sense for any finite graph $(G, E)$ and is called the uniform spanning tree; it will be encountered again in Section 3.

Given a random forest $\mu$ on a group $G$, we denote by $f_{\mu}(g)$ the probability that $g \in G$ is neighboring the identity $1 \in G$. In other words, $f_{\mu}(g)=\mu\left\{F \in \mathscr{F}_{G}:(1, g) \in F\right\}$. If $\mu$ has finite width, then $f_{\mu}$ is a finitely supported function.

We now recall the definition of the $T^{1}$-norm on the space $\mathrm{C}[G]$ of finitely supported functions. Given $f \in \mathbf{C}[G]$, one considers all pairs $f^{ \pm}$of functions $G \times G \rightarrow \mathbf{C}$ such that

$$
f\left(g^{-1} g^{\prime}\right)=f^{+}\left(g, g^{\prime}\right)+f^{-}\left(g, g^{\prime}\right) \quad \forall g, g^{\prime} \in G
$$

The norm $\|f\|_{T^{1}(G)}$ is the infimum over all such pairs $f^{ \pm}$of the expression

$$
\sup _{g \in G} \sum_{g^{\prime} \in G}\left|f^{+}\left(g, g^{\prime}\right)\right|+\sup _{g \in G} \sum_{g^{\prime} \in G}\left|f^{-}\left(g^{\prime}, g\right)\right| .
$$

The completion of $\mathbf{C}[G]$ for this norm is a Banach space denoted by $T^{1}(G)$ that can be realized as functions on $G$; we refer to [43] for details and context. Such functions are called Littlewood functions (see e.g. $[6,8,50]$ ) in reference to classical harmonic analysis [32].

Proposition 2.3. Let $\mu$ be a random forest of finite width on a countable group. Then

$$
\left\|f_{\mu}\right\|_{T^{1}(G)} \leq 2 \quad \text { and } \quad\left\|f_{\mu}\right\|_{\ell^{2}(G)} \geq \frac{\operatorname{deg}(\mu)}{\sqrt{\operatorname{width}(\mu)}}
$$


This proposition is a concrete way to carry over to random forests the geometric aspects of a construction for free groups from [6], in accordance with the ideas expressed in the Introduction.

Proof of Proposition 2.3. The second inequality is a straightforward application of the Cauchy-Schwarz inequality: setting $S=\left\{g: f_{\mu}(g)>0\right\}$, we have $|S|=\operatorname{width}(\mu)$ and hence

$$
\operatorname{deg}(\mu)=\sum_{g \in G} f_{\mu}(g)=\sum_{g \in G} f_{\mu}(g) \cdot 1_{S}(g) \leq \sqrt{\operatorname{width}(\mu)}\left\|f_{\mu}\right\|_{\ell^{2}(G)},
$$

as claimed.

We now focus on the first inequality. Let $\left\{g_{n}\right\}_{n \in \mathbf{N}}$ be an enumeration of the group $G$. We define a Borel section $\mathscr{O}: \mathscr{F}_{G} \rightarrow \mathscr{F}_{G}^{+}$as follows. For a forest $F$ and $\left(g, g^{\prime}\right) \in F$, let $n$ be the first integer such that $g_{n}$ belongs to the tree containing $\left(g, g^{\prime}\right)$. We then declare that $\left(g, g^{\prime}\right)$ belongs to $\mathscr{O}(F)$ if $g^{\prime}$ lies between $g$ and $g_{n}$ in that tree; otherwise, $\left(g^{\prime}, g\right) \in \mathscr{O}(F)$.

We now define two functions $f_{\mu}^{ \pm}$on $G \times G$ by

$$
f_{\mu}^{+}\left(g, g^{\prime}\right)=\mu\left\{F \in \mathscr{F}_{G}:\left(g, g^{\prime}\right) \in \mathscr{O}(F)\right\}, \quad f_{\mu}^{-}\left(g, g^{\prime}\right)=f_{\mu}^{+}\left(g^{\prime}, g\right)
$$

The sum $f_{\mu}^{+}\left(g, g^{\prime}\right)+f_{\mu}^{-}\left(g, g^{\prime}\right)$ is $\mu\left\{F \in \mathscr{F}_{G}:\left(g, g^{\prime}\right) \in F\right\}$ by the definition of an orientation. Since $\mu$ is $G$-invariant, this quantity depends only on $g^{-1} g^{\prime}$ and thus coincides with $f_{\mu}\left(g^{-1} g^{\prime}\right)$. Therefore, in view of the definition of $T^{1}(G)$, it remains to justify

$$
\sup _{g \in G} \sum_{g^{\prime} \in G} f_{\mu}^{+}\left(g, g^{\prime}\right) \leq 1
$$

Thus, fix any $g \in G$. Given a forest $F$, there is at most one $g^{\prime} \in G$ such that $\left(g, g^{\prime}\right) \in \mathscr{O}(F)$. Indeed, the integer $n$ introduced in the definition of $\mathscr{O}$ is uniquely determined by $g$ and thus $g^{\prime}$ can only be the first step towards $g_{n}$ from $g$, unless $g=g_{n}$ in which case there is no such $g^{\prime}$. Therefore, $\sum_{g^{\prime}} f_{\mu}^{+}\left(g, g^{\prime}\right)$ is a sum of measures of disjoint subsets of $\mathscr{F}_{G}$ and hence is bounded by $\mu\left(\mathscr{F}_{G}\right)=1$.

The space $T^{1}(G)$ is directly related to uniformly bounded representations.

Proposition 2.4. If $G$ is unitarizable, then there is a constant $K$ such that

$$
\|\cdot\|_{\ell^{2}(H)} \leq K\|\cdot\|_{T^{1}(H)}
$$

holds for all subgroups $H<G$. 
Observe that the juxtaposition of Propositions 2.3 and 2.4 establishes Theorem 1.3.

Proof of Proposition 2.4. The fact that unitarizability implies $T^{1}(G) \subseteq \ell^{2}(G)$ was established in [6, 2.3(i)], see also Remark 2.8 in [43]. We sketch the main idea for convenience. First, any $T^{1}$-function gives rise to a uniformly bounded representation on $\ell^{2}(G) \oplus \ell^{2}(G)$ by twisting the (diagonal) regular representation with the derivation given by the commutator between the regular representation and kernel operator defined by $f^{+}$(using $f^{-}$ yields the same derivation up to a sign since $f^{+}+f^{-}$is $G$-invariant). If $G$ is unitarizable, this construction implies that $T^{1}(G)$ is contained in the space $B(G)$ of matrix coefficients of unitary representations on $G$. Then the stronger conclusion $T^{1}(G) \subseteq \ell^{2}(G)$ is obtained by a cotype argument.

Next, we claim that this inclusion is continuous. This follows from the closed graph theorem; indeed, the diagonal in $T^{1}(G) \times \ell^{2}(G)$ is closed since it is closed for the weaker topology of pointwise convergence (the latter being Hausdorff).

To conclude the proof, it suffices to show that for all subgroups $H<G$ the canonical inclusion map $\mathbf{C}[H] \rightarrow \mathbf{C}[G]$ extends to an isometric map $T^{1}(H) \rightarrow T^{1}(G)$ since the analogous statement for $\ell^{2}(H) \rightarrow \ell^{2}(G)$ is obvious. Following [43, 2.7(ii)], we choose a set $R \subseteq G$ of representatives for $G / H$; we arrange that $R$ contains the identity. Given $f \in T^{1}(H)$, we still write $f: G \rightarrow \mathrm{C}$ for the function extended by zero outside $H$. Let $f^{ \pm}$ be any pair of functions $H \times H \rightarrow \mathrm{C}$ as required by the definition of $T^{1}(H)$. We now extend the definition of $f^{ \pm}$to functions $G \times G \rightarrow \mathrm{C}$ by setting

$$
f^{ \pm}\left(g, g^{\prime}\right)= \begin{cases}f^{ \pm}\left(h, h^{\prime}\right) & \text { if } g=r h, g^{\prime}=r h^{\prime} \text { for } r \in R \text { and } h, h^{\prime} \in H \\ 0 & \text { otherwise. }\end{cases}
$$

The definition is well posed since $R$ maps injectively to $G / H$. This construction witnesses that $f \in T^{1}(G)$ with $T^{1}(G)$-norm bounded by $\|f\|_{T^{1}(H)}$; the reverse inequality is immediate.

\section{First $L^{2}$-Betti Number}

To achieve this wonder, electricity is the one and only means. Inestimable good has already been done by the use of this all powerful agent, the nature of which is still a mystery.

(N. Tesla, The transmission of electrical energy without wires as a means for furthering Peace, 1905.) 
In 1847, Kirchhoff [26] proved that given a unit electric current between the endpoints of an edge $e$ in a finite graph, the current flowing through $e$ equals the (counting) probability that $e$ belongs to the uniform spanning tree as introduced in Example 2.2. There is a well-known connection between currents and combinatorially harmonic functions (see Weyl [51] or Eckmann [14] and [13], pp. 247-8). This is the starting point for the relation between random forests and the first $L^{2}$-Betti number that emerged from the work of Pemantle [42], Gaboriau [21], and Lyons, exposed in [33]. We shall present just what we need in our setting and refer to [7] and [33] for more material.

Let $H$ be a countable group and $S \subseteq H$ some finite subset. Consider the graph $\mathfrak{g}=$ $(H, E)$ obtained by assigning a geometric edge (i.e. two opposed elements of $E$ ) between $h, h^{\prime} \in H$ whenever $h^{-1} h^{\prime}$ is in $S \cup S^{-1}$. Recall that when $S$ generates $H$, the graph $\mathfrak{g}$ is called a Cayley graph for $H$. The left $H$-action preserves the graph structure, and we shall investigate random forests arising as subgraphs of $\mathfrak{g}$. Given an enumeration of $H$, let $\mathfrak{g}_{n}$ be the subgraph of $\mathfrak{g}$ spanned by the first $n$ elements in $H$. Pemantle [42] showed that the uniform spanning tree measure on $\mathfrak{g}_{n}$ converges weakly to a measure on $\mathscr{F}_{H}$. Indeed, it suffices essentially to prove that the probability of the elementary event that a given edge $e$ belongs to a tree in $\mathfrak{g}_{n}$ (with $n$ large enough to ensure $e \in \mathfrak{g}_{n}$ ) is nonincreasing in $n$. In view of Kirchhoff's result, this monotonicity follows from Rayleigh's principle stating that added edges can only reduce the current through a given edge. The resulting measure on the space of subgraphs is supported on $\mathscr{F}_{G}$ since the latter is closed; it is called the free uniform spanning forest. (Notice that finite trees can and generally do get disconnected in the limit.) The monotonicity implies in particular that the limit measure does not depend on the enumeration and hence is group-invariant. More information about this measure can be found in [7, 33, 42].

Let $\ell_{\text {alt }}^{2}(\mathfrak{g})$ be the space of square-summable functions on $E$ that change sign under the involution $e \mapsto \bar{e}$ (i.e. "1-forms"), endowed with the Hilbertian structure induced by the inclusion $\ell_{\text {alt }}^{2}(\mathfrak{g}) \subseteq \ell^{2}(E)$. Define the elementary edge function $\chi_{e}:=\delta_{e}-\delta_{\bar{e}}$, where $\delta$ is the Dirac mass. Denote by $d: \ell^{2}(H) \rightarrow \ell_{\text {alt }}^{2}(\mathfrak{g})$ the combinatorial derivative (coboundary) defined by $d f(e)=f\left(e_{+}\right)-f\left(e_{-}\right)$and by $d^{*}$ its adjoint. Let $\ell_{\star}^{2}(\mathfrak{g}) \subseteq \ell_{\text {alt }}^{2}(\mathfrak{g})$ be the closure of $d \ell^{2}(H)$ and $\ell_{\bigcirc}^{2}(\mathfrak{g}) \subseteq \ell_{\text {alt }}^{2}(\mathfrak{g})$ the closed span of all cycles (i.e. sums $\sum_{i} \chi_{e_{i}}$ for sequences $\left\{e_{i}\right\}$ forming cycles). We make the corresponding definitions for the graphs $\mathfrak{g}_{n}$. The latter being finite, linear algebra provides the orthogonal decomposition $\ell_{\text {alt }}^{2}\left(\mathfrak{g}_{n}\right)=\ell_{\star}^{2}\left(\mathfrak{g}_{n}\right) \oplus \ell_{\bigcirc}^{2}\left(\mathfrak{g}_{n}\right)$. The failure of this relation for a general infinite graph $\mathfrak{g}$ is crucial below. Equally important is the fact that while $\ell_{\text {alt }}^{2}\left(\mathfrak{g}_{n}\right)$ and $\ell_{\bigcirc}^{2}\left(\mathfrak{g}_{n}\right)$ clearly densely exhaust $\ell_{\text {alt }}^{2}(\mathfrak{g})$ and $\ell_{\bigcirc}^{2}(\mathfrak{g})$ as $n \rightarrow \infty$, the corresponding circumstance does not hold for $\ell_{\star}^{2}\left(\mathfrak{g}_{n}\right)$, which is not even a subspace of $\ell_{\star}^{2}(\mathfrak{g})$. (This is a key difference between the present model of free 
random forests and the so-called wired case where the finite approximations $\mathfrak{g}_{n}$ are defined differently.)

We record the following result stated (with all necessary indications for the proof) in the current version of Chapter 10 of the book in progress [33].

Proposition 3.1. If $S$ generates $H$, then the expected degree of the free uniform spanning forest is at least $2 \beta^{1}(H)$.

In fact, the exact value $2 \beta^{1}(H)+2$ is given in [33] (compare Remark 3.2).

Proof of Proposition 3.1. We can assume $S=S^{-1}$ and $1 \notin S$ without affecting the statement, so that the neighbors of 1 in $\mathfrak{g}=(H, E)$ are exactly $S$. Given an edge $e$ and $n$ large enough, denote by $i_{n}(e)$ the probability that $e$ (or rather the corresponding geometric edge) is in the uniform spanning tree of $\mathfrak{g}_{n}$. We need to prove

$$
\sum_{s \in S} \lim _{n \rightarrow \infty} i_{n}\left(e_{s}\right) \geq 2 \beta^{1}(H), \quad \text { wherein } \quad e_{s}:=(s, 1) .
$$

By definition, the first $L^{2}$-Betti number $\beta^{1}(H)$ is the von Neumann dimension of the first $L^{2}$-cohomology of $H$. The dimension is not affected by passing to the Hausdorff quotient called the reduced $L^{2}$-cohomology. The latter admits a Hodge-de Rham decomposition which realizes the first reduced $L^{2}$-cohomology of the finitely generated group $H$ as the space

$$
\mathscr{D}_{\mathfrak{g}}:=\left(\ell_{\star}^{2}(\mathfrak{g}) \oplus \ell_{\bigcirc}^{2}(\mathfrak{g})\right)^{\perp} \subseteq \ell_{\text {alt }}^{2}(\mathfrak{g})
$$

of coboundaries of harmonic functions on vertices [37, Section 1.1.4]. (In other words $\mathscr{D}_{\mathfrak{g}}$ is the space of differentials of harmonic Dirichlet functions, which is isomorphic to the quotient of harmonic Dirichlet functions by the constants.) As for the von Neumann dimension, we recall that for a closed invariant subspace $W<\ell^{2}(H)$ it is given explicitly by $\pi_{W}\left(\delta_{1}\right)(1)$, where $\pi_{W}: \ell^{2}(H) \rightarrow W$ is the orthogonal projection. Combining this with the canonical isometric $H$-identification $\ell_{\text {alt }}^{2}(\mathfrak{g}) \cong \oplus_{s \in S} \ell^{2}\left(H \cdot e_{s}\right)$ determined by $\chi_{e_{s}} \mapsto 2 \delta_{e_{s}}$, one has

$$
\beta^{1}(H)=\frac{1}{2} \sum_{s \in S} \pi_{\mathscr{D}_{\mathfrak{g}}}\left(\chi_{e_{s}}\right)\left(e_{S}\right),
$$

where $\pi_{\mathscr{D}_{\mathfrak{g}}}: \ell_{\text {alt }}^{2}(\mathfrak{g}) \rightarrow \mathscr{D}_{\mathfrak{g}}$. A hurried reader may as well skip the above paragraph and take this identity as ad hoc definition of $\beta^{1}$.

In view of Kirchhoff's laws, the current on $\mathfrak{g}_{n}$ yielding unit flow between the endpoints of an edge $e$ is $\pi_{\ell_{\star}^{2}\left(\mathfrak{g}_{n}\right)}\left(\chi_{e}\right)$ (see e.g. [13], p. 248). Therefore, Kirchhoff's 
characterization [26] in terms of the uniform spanning tree shows $i_{n}(e)=\pi_{\ell_{\star}^{2}\left(\mathfrak{g}_{n}\right)}\left(\chi_{e}\right)(e)$. Recalling that $\ell_{\bigcirc}^{2}$, but not $\ell_{\star}^{2}$, is compatible with the exhaustion, we obtain

$$
\begin{aligned}
\sum_{s \in S} \lim _{n \rightarrow \infty} i_{n}\left(e_{S}\right) & =\sum_{s \in S} \pi_{\ell_{\bigcirc}^{2}(\mathfrak{g})^{\perp}}\left(\chi_{e_{s}}\right)\left(e_{s}\right)=\sum_{s \in S} \pi_{\mathscr{D}_{\mathfrak{g}} \oplus \ell_{\star}^{2}(\mathfrak{g})}\left(\chi_{e_{s}}\right)\left(e_{s}\right) \\
& =\sum_{s \in S} \pi_{\mathscr{D}_{\mathfrak{g}}}\left(\chi_{e_{s}}\right)\left(e_{s}\right)+\sum_{s \in S} \pi_{\ell_{\star}^{2}(\mathfrak{g})}\left(\chi_{e_{s}}\right)\left(e_{s}\right) .
\end{aligned}
$$

We know already that the first summand equals $2 \beta^{1}(H)$. In order to conclude the proof, it remains only to justify that the function $f:=\pi_{\ell_{\star}^{2}(\mathfrak{g})}\left(\chi_{e_{s}}\right)$ is nonnegative at $e_{s}$. This is the case since (i) $\chi_{e_{s}}\left(e_{s}\right)=1$, (ii) $f$ and $\chi_{e_{s}}$ are alternating, and (iii) orthogonality imposes $\left\|f-\chi_{e_{s}}\right\| \leq\left\|\chi_{e_{s}}\right\|=\sqrt{2}$.

Remark 3.2. The expected degree is $2 \beta^{1}(H)+2$. Indeed, the second summand in the proof above is the expected degree of the wired uniform spanning forest on $\mathfrak{g}$ for reasons entirely similar to the above, namely because the exhaustion defining this other model is compatible with $\ell_{\star}^{2}$. On the other hand, it is shown in [7] that this expected degree is two, using a different characterization of the wired forest via an algorithm of Wilson [52].

We are now ready to complete the reduction of Theorem 1.1 to Theorem 1.3. Recall that the $\operatorname{rank} \operatorname{rk}(H)$ is the minimal number of generators of a group $H$.

Proof of Theorem 1.5, first bound. Let $H<G$ be a finitely generated subgroup with a generating set $S$ of size $\operatorname{rk}(H)$. The corresponding free uniform spanning forest $\mu$ on $H$ satisfies width $(\mu) \leq \operatorname{rk}(H)$. Therefore, Proposition 3.1 shows that Theorem 1.3 yields the desired bound.

Proof of Theorem 1.1. Let $G$ be any residually finite group with $\beta^{1}(G)>0$. Since $G$ is the union of the directed set of all its finitely generated subgroups, Theorem 7.2(3) in [37] provides us with a finitely generated subgroup $G_{0}<G$ with $\beta^{1}\left(G_{0}\right)>0$. Strictly speaking, one needs to express $G$ as a directed union of infinite subgroups in order to apply Theorem 7.2(3) in [37]; this is not a restriction since if no such family existed, then $G$ would be amenable as directed union of finite groups, contradicting $\beta^{1}(G)>0$ (Theorem 0.2 in [10]).

The group $G_{0}$ is still residually finite; we shall use the weaker property that $G_{0}$ admits finite index subgroups of arbitrarily large index. Notice that for all finite index subgroups $H<G_{0}$, the quantities $\operatorname{rk}(H)$ and $\beta^{1}(H)$ are finite. Moreover, denoting 
by $\left[G_{0}: H\right]$ the index, one has

$$
\beta^{1}(H)=\left[G_{0}: H\right] \beta^{1}\left(G_{0}\right) \quad \text { and } \quad \operatorname{rk}(H) \leq\left[G_{0}: H\right] \operatorname{rk}\left(G_{0}\right)
$$

The above equality is a basic property of $L^{2}$-Betti numbers [37, 1.35(9)] while the inequality is a (nonoptimal) consequence of the Reidemeister-Schreier algorithm, see e.g. Proposition 4.1 of [36] (in fact the quantity rk - 1 is submultiplicative).

Since $\left[G_{0}: H\right]$ is unbounded, the above (in)equalities violate the first bound of Theorem 1.5.

\section{Cost}

This section will be more concise since we shall deduce Theorem 1.2 from Theorem 1.3 in very much the same way as we did for Theorem 1.1. We refer to Section 5 for further comments.

The $\operatorname{cost} \mathscr{C}(G)$ of a countable group $G$ is a numerical invariant extensively studied by Gaboriau [18] (and suggested by Levitt [31, p. 1174]). It is defined as the infimum over all free probability-preserving $G$-actions and over all families of partial isomorphisms generating the resulting orbit equivalence relation of the sum of the measures of the domains of the partial isomorphisms.

We shall not use this definition, but rather the following alternative definition. The cost $\mathscr{C}(G)$ is the infimum of half the expected degree over all connected random graphings of $G$. The equivalence of the definitions is proved in Proposition 29.5 of [27] (where the definition of the degree differs by a factor 2).

We now proceed to recall another family of models of random forests, namely the free minimal spanning forests; first studied on $\mathbf{Z}^{d}$ in $[2,3]$, it received a general treatment in [34]. Let $H$ be a group generated by a finite set $S=S^{-1} \not \supset 1$, and let $\mathfrak{g}$ be the corresponding Cayley graph $\mathfrak{g}=(H, E)$ (as in Section 3). The free minimal spanning forest associated to this choice $\mathfrak{g}$ is the random graphing of $H$ obtained by assigning weights on the (geometric) edges of $\mathfrak{g}$ independently and deleting every edge that has maximal weight in some cycle. We shall need the following fact due to Lyons.

Proposition 4.1. Let $\mu$ be the above random forest. Then $\operatorname{deg}(\mu) \geq 2 \mathscr{C}(H)$.

Proof. For any $0 \leq p \leq 1$, let $\mu_{p}$ be the random graphing obtained by adding to the $\mu$ random forest each edge of $E$ with probability $p$, independently (thus $\mu_{p}$ is the union of $\mu$ and of the Bernoulli $p$-percolation random graphing on $\mathfrak{g}$ ). According to Theorem 1.3 
in [34], $\mu_{p}$ is almost surely connected whenever $p>0$. However, we have by construction

$$
\operatorname{deg}\left(\mu_{p}\right) \leq \operatorname{deg}(\mu)+|S| \cdot p .
$$

Letting $p$ tend to zero, the statement follows from the characterization of $\mathscr{C}(H)$ recalled above.

Now the reduction of Theorem 1.2 to Theorem 1.3 proceeds exactly along the lines of the arguments given for Theorem 1.1 in Section 3. First, Proposition 4.1 applied to finitely generated subgroups $H$ of $G$ shows that Theorem 1.3 yields the second bound of Theorem 1.5. Then, one considers finite index subgroups $H<G$ of arbitrarily large index and argues as for Theorem 1.1, using this time the relation

$$
\mathscr{C}(H)-1=[G: H](\mathscr{C}(G)-1),
$$

which is Theorem 3 in [18]. There is no need to choose a subgroup $G_{0}$ since $G$ was assumed finitely generated from the outset.

It was pointed out by a referee that one could replace the explicit model of the minimal spanning forest used above by the following argument. Given a free probabilitypreserving action of $G$ on some $(X, \mu)$, one can prove that the corresponding equivalence relation on $X$ contains a measurable subrelation admitting an acyclic graphing of no lesser cost (see [27, 28.11]); moreover, this graphing can be chosen to be supported on the graphing produced by a Cayley graph of $G$. The measure pushforward argument used in the proof of $[27,29.5]$ provides a random forest on the given Cayley graph. This provides an alternative to Proposition 4.1, the remaining arguments remaining unchanged.

\section{Further Considerations}

\section{1}

The second bound of Theorem 1.5 implies the first. Indeed, for any infinite countable group $G$, one has $\mathscr{C}(G)-1 \geq \beta^{1}(G)$ (this follows from Corollary 3.23 in [19]). A well-known question is whether equality holds. Thus, for finitely generated groups, Theorem 1.2 is a priori stronger than Theorem 1.1. For countable groups, one would need the fact that a directed union of cost one groups still has cost one; this is not in the literature, but Gaboriau and a referee communicated the following proof. Suppose $G$ is the increasing union of subgroups $G_{n}$ admitting free cost one actions on $X_{n}$. The diagonal $G$-action on the product of the coinduced actions $X=\prod X_{n}^{G / G_{n}}$, when restricted to $G_{n}$, admits 
$G_{n}$-action on $X_{n}$ as a factor (see [20, Section 3.4]). Thus, the $G_{n}$-action on $X$ has cost one (see e.g. [25], last remark in Section 10). It follows that the $G$-action on $X$ also has cost one (see Lemma 6.25 in [18]).

As for the two types of forests used on finitely generated groups in the reduction of these two theorems to Theorem 1.3, it is a general fact that on the same Cayley graph, the free minimal spanning forest has expected degree bounded below by its uniform analog (see Corollary 1.4 in [34]).

\section{2}

As mentioned in the Introduction, any nonamenable finitely generated group $G$ admits a random forest of expected degree $>2$. Indeed, let $S=S^{-1} \not \supset 1$ a finite generating set. For an integer $k$, consider the $k$ th product graph $\mathfrak{g}^{[k]}$ associated to the Cayley graph $\mathfrak{g}$, recalling that it consists of the graph on $G$ where edges correspond to $k$-paths in $\mathfrak{g}$. Strictly speaking, it is a multigraph, but any forest on $\mathfrak{g}^{[k]}$ can be considered as a forest on the Cayley graph associated to $S^{k}$. Using spectral isoperimetric estimates, it is proved in [46] that the Bernoulli percolation on $\mathfrak{g}^{[k]}$ satisfies $p_{c}<p_{u}$ when $k$ is large enough, where $p_{c}, p_{u}$ are, respectively, the critical probability and the uniqueness probability (see [33] for more background). By Proposition 1.7 in [34], this implies that the free minimal spanning forest differs from its wired analog, which implies that the former has higher expected degree by Proposition 3.5 in [34]. We recall here that the wired minimal forest is defined exactly as in Section 4, except that one deletes an edge if it has maximal weight even in a cycle "through infinity," which is just a bi-infinite path (our reference is still [34]). Summing up, it remains only to prove that the expected degree of the wired minimal spanning forest is at least 2 . In fact, it is exactly 2 in the Cayley graph case at hand (see Theorem 3.12 in [34]). The above reasoning can be extracted from the arguments of [22].

\section{3}

It would be desirable to have examples of residually finite groups $G$ with $\beta^{1}(G)>0$ or $\mathscr{C}(G)>1$, but not containing $F_{2}$. We would expect such examples to exist, be it only because the nonvanishing of $\beta^{1}$ is a measure equivalence invariant by [19], and $\mathscr{C}>1$ is so by definition; it seems unlikely that the containment of $F_{2}$ should be preserved. Interestingly, it is established in [47] that for a torsion-free group satisfying a weaker form of the Atiyah conjecture, $\beta^{1}>0$ implies the existence of a free subgroup $F_{2}$. In 
view of the measure equivalence invariance of $\beta^{1}>0$, one can ask if this statement should be considered as evidence against the Atiyah conjecture. On the other hand, an indication of perhaps surprisingly strong restrictions given by additional algebraic assumptions is Lackenby's result [29] that implies, in particular, that residually p-finite finitely presented groups with $\beta^{1}>0$ contain $F_{2}$.

5.4

Let $G$ be a group generated by a finite set $S$, and let $\mathfrak{g}$ be the corresponding Cayley graph. Theorem 1.3 is an incentive to find random forests in $\mathfrak{g}$ with large expected degree (compared to the size of $S$ ). One immediate restriction is given by the vertex isoperimetric constant of $\mathfrak{g}$, namely the infimum $i_{V}(\mathfrak{g})$ of the ratio $\left|\partial_{V} \mathfrak{h}\right| /|\mathfrak{h}|$, where $\mathfrak{h}$ ranges over all finite subgraphs and $\partial_{V}$ denotes the vertex boundary. Indeed, one verifies that the expected degree of any random forest on $\mathfrak{g}$ is bounded by $1+i_{V}(\mathfrak{g}) / 2$. (For the edge-isoperimetric constant, this inequality occurs in [35].) One can increase at will $i_{V}$ for any nonamenable graph by replacing $S$ with high powers of that set (as in [46], see Section 5.2 above), but this procedure also affects the denominator in Theorem 1.3.

While an application of the Hall marriage lemma and of a Cantor-Bernstein argument shows that $\mathfrak{g}$ contains a forest of $n$-regular trees whenever $n \leq i_{V}(\mathfrak{g}$ ) (see also Theorem 1.2 in [9]), there is no indication that there should be a $G$-invariant measure on the space of such forests.

\section{5}

Let $G$ be a finitely generated group. Rather than residual finiteness, the proof of Theorem 1.1 (and thus also of Theorem 1.2) actually uses the existence of infinitely many finite quotients of $G$ or equivalently of some infinite sequence $\left\{H_{n}\right\}$ of finite index subgroups $H_{n}<G$, which we may assume nested. The Reidemeister-Schreier algorithm quoted earlier shows that the limit

$$
\lim _{n \rightarrow \infty} \frac{\operatorname{rk}\left(H_{n}\right)-1}{\left[G: H_{n}\right]}
$$

exists; it was introduced in [30] as the rank gradient. The absolute rank gradient of [5] is the infimum of the above ratio over all finite index subgroups of $G$.

Does the existence of an infinite sequence with positive rank gradient imply that there are random forests $\mu$ on (subgroups of) $G$ with unbounded ratio $\operatorname{deg}(\mu)^{2} / \operatorname{width}(\mu)$ ? Are there such forests at least when $G$ has positive absolute rank gradient? 
It follows from the definitions that both $\beta^{1}(G)$ and $\mathscr{C}(G)$ are bounded by $\operatorname{rk}(G)$; therefore, the multiplicativity of $\beta^{1}$ and $\mathscr{C}$ (as recalled in earlier sections) implies that both are lower bounds for the absolute rank gradient. The results of Abért-Nikolov [4] suggest some similarity of the rank gradient with the behavior of these invariants. Moreover, in [5], Abért-Nikolov express the rank gradient of certain chains $\left\{H_{n}\right\}$ as the cost of a specific $G$-action attached to the chain. This result gives added interest to the fixed price question which asks whether all relations produced by a given countable group have same cost [18].

\section{Acknowledgments}

It is a pleasure to thank the following colleagues: Gilles Pisier for first mentioning Dixmier's problem to us; Adrian Ioana for finding a mistake in an earlier draft; and Wolfgang Lück for helping out with a reference. Special thanks to Miklós Abért for suggesting to use the cost and to Denis Osin for providing the groups mentioned in Corollary 1.6. The anonymous referees provided very helpful comments. I.E. was supported in part by the NSF and the Clay Institute and N.M. was supported in part by the Swiss National Science Foundation.

\section{References}

[1] Adyan, S. I. "Random walks on free periodic groups." Izvestiya Akademii Nauk SSSR Seriya Matematicheskaya 46, no. 6 (1982): 1139-49.

[2] Alexander, K. S. "Percolation and minimal spanning forests in infinite graphs." Annals of Probability 23, no. 1 (1995): 87-104.

[3] Alexander, K. S., and S. A. Molchanov. "Percolation of level sets for two-dimensional random fields with lattice symmetry." Journal of Statistical Physics 77, nos. 3-4 (1994): 627-43.

[4] Abért, M., A. Jaikin-Zapirain, and N. Nikolov. "The rank gradient from a combinatorial viewpoint." Preprint available at: http://arxiv.org/abs/math/0701925v2.

[5] Abért, M., and N. Nikolov. "Rank gradient, cost of groups and the rank versus Heegaard genus problem." Preprint available at: http://arxiv.org/abs/math/0701361v3.

[6] Bożejko, M., and G. Fendler. "Herz-Schur multipliers and uniformly bounded representations of discrete groups." Archiv der Mathematik 57, no. 3 (1991): 290-8.

[7] Benjamini, I., R. Lyons, Y. Peres, and O. Schramm. "Uniform spanning forests." Annals of Probability 29, no. 1 (2001): 1-65.

[8] Bożejko, M. "Uniformly bounded representations of free groups." Journal für die Reine und Angewandte Mathematik 377 (1987): 170-86.

[9] Benjamini, I., and O. Schramm. "Every graph with a positive Cheeger constant contains a tree with a positive Cheeger constant." Geometric and Functional Analysis 7, no. 3 (1997): 403-19. 
[10] Cheeger, J., and M. Gromov. " $L_{2}$-cohomology and group cohomology." Topology 25, no. 2 (1986): 189-215.

[11] Day, M. M. "Means for the bounded functions and ergodicity of the bounded representations of semigroups." Transactions of the American Mathematical Society 69 (1950): 276-91.

[12] Dixmier, J. "Les moyennes invariantes dans les semi-groupes et leurs applications." Acta University Szegediensis Scientiarum Mathematicarum 12 (1950): 213-27.

[13] Eckmann, B. "Harmonische Funktionen und Randwertaufgaben in einem Komplex." Commentarii Mathematici Helvetici 17 (1945): 240-55.

[14] Eckmann, B. "Lois de Kirchhoff et fonctions discrètes harmoniques." Bulletin de la Société Vaudoise des Sciences Naturelles 63 (1945): 67-78.

[15] Eckmann, B. "Introduction to $\ell_{2}$-methods in topology: Reduced $\ell_{2}$-homology, harmonic chains, $\ell_{2}$-Betti numbers." Israel Journal of Mathematics 117 (2000): 183-219.

[16] Ehrenpreis, L., and F. I. Mautner. "Uniformly bounded representations of groups." Proceedings of the National Academy of Sciences of the United States of America 41 (1955): 231-3.

[17] Epstein, I. “Orbit inequivalent actions of nonamenable groups." (2007): preprint arXiv.org:0707.4215v2.

[18] Gaboriau, D. "Coût des relations d'équivalence et des groupes." Inventiones Mathematicae 139, no. 1 (2000): 41-98.

[19] Gaboriau, D. "Invariants $l^{2}$ de relations d'équivalence et de groupes." Publications Mathématiques Institut des Hautes Études Scientifiques 95 (2002): 93-150.

[20] Gaboriau, D. "Examples of groups that are measure equivalent to the free group." Ergodic Theory and Dynamical Systems 25, no. 6 (2005): 1809-27.

[21] Gaboriau, D. "Invariant percolation and harmonic Dirichlet functions." Geometric and Functional Analysis 15, no. 5 (2005): 1004-51.

[22] Gaboriau, D., and R. Lyons. “A measurable-group-theoretic solution to von Neumann's problem." Inventiones Mathematicae (2007): preprint arXiv.org:0711.1643.

[23] Hjorth, G. "A lemma for cost attained." Annals of Pure and Applied Logic 143, nos. 1-3 (2006): 87-102.

[24] Johnson, B. E. Cohomology in Banach Algebras. Memoirs of the American Mathematical Society 127. Providence, RI: American Mathematical Society, 1972.

[25] Kechris, A. S. Global Aspects of Ergodic Group Actions. Mathematical Surveys and Monographs (forthcoming).

[26] Kirchhoff, G. “Ueber die Auflösung der Gleichungen, auf welche man bei der Untersuchung der linearen Vertheilung galvanischer Ströme geführt wird." Annalen der Physik und Chemie 72, no. 12 (1847): 497-508.

[27] Kechris, A. S., and B. D. Miller. Topics in Orbit Equivalence. Lecture Notes in Mathematics 1852. Berlin: Springer, 2004.

[28] Kunze, R. A., and E. M. Stein. "Uniformly bounded representations and harmonic analysis of the $2 \times 2$ real unimodular group." American Journal of Mathematics 82 (1960): 1-62.

[29] Lackenby, M. "Detecting large groups." Preprint available at: http://arxiv.org/abs/math/ $0702571 \mathrm{v} 1$. 
[30] Lackenby, M. "Expanders, rank and graphs of groups." Israel Journal of Mathematics 146 (2005): 357-70.

[31] Levitt, G. “On the cost of generating an equivalence relation." Ergodic Theory and Dynamical Systems 15, no. 6 (1995): 1173-81.

[32] Littlewood, J. E. "On bounded bilinear forms in an infinite number of variables." Quarterly Journal of Mathematics 1 (1930): 164-74.

[33] Lyons, R., and Y. Peres. "Probability on trees and networks." Preprint available at: http://mypage.iu.edu/ rdlyons/prbtree/prbtree.html.

[34] Lyons, R., Y. Peres, and O. Schramm. "Minimal spanning forests." Annals of Probability 34, no. 5 (2006): 1665-92.

[35] Lyons, R., M. Pichot, and S. Vassout. "Uniform nonamenability, cost, and the first $\ell^{2}$-Betti number." Geometry, Groups, and Dynamics 2 (2008): 595-617.

[36] Lyndon, R. C., and P. E. Schupp. Combinatorial Group Theory. Ergebnisse der Mathematik und ihrer Grenzgebiete, Band 89. Berlin: Springer, 1977.

[37] Lück, W. $L^{2}$-Invariants: Theory and Applications to Geometry and K-Theory. Ergebnisse der Mathematik und ihrer Grenzgebiete 3, Folge A. Series of Modern Surveys in Mathematics 44. Berlin: Springer, 2002.

[38] Monod, N. "An invitation to bounded cohomology." In Proceedings of the International Congress of Mathematicians, vol. 2, 1183-211. Invited Lectures. Zürich: European Mathematical Society, 2006.

[39] Mantero, A. M., and A. Zappa. "Uniformly bounded representations and $L^{p}$-convolution operators on a free group." In Harmonic Analysis, 333-43. Lecture Notes in Mathematics 992. Berlin: Springer, 1983.

[40] Ol'shanskii, A. Y. “On the question of the existence of an invariant mean on a group." Uspekhi Matematicheskikh Nauk 35, no. 4 (1980): 199-200.

[41] Osin, O. " $L^{2}$-Betti numbers and non-unitarizable groups without free subgroups." (2008): preprint available from http://front.math.ucdavis.edu/0812.2093.

[42] Pemantle, R. "Choosing a spanning tree for the integer lattice uniformly." Annals of Probability 19, no. 4 (1991): 1559-74.

[43] Pisier, G. Similarity Problems and Completely Bounded Maps, 2nd expanded ed. Lecture Notes in Mathematics 1618. Berlin: Springer, 2001.

[44] Pisier, G. “Are unitarizable groups amenable?" In Infinite Groups: Geometric, Combinatorial and Dynamical Aspects, 323-62. Progress in Mathematics 248. Basel: Birkhäuser, 2005.

[45] Pytlik, T., and R. Szwarc. "An analytic family of uniformly bounded representations of free groups." Acta Mathematica 157 (1986): 287-309.

[46] Pak, I., and T. Smirnova-Nagnibeda. "On nonuniqueness of percolation on nonamenable Cayley graphs." Comptes Rendus de l'Académie des Sciences Série 1 Mathématiques 330, no. 6 (2000): 495-500.

[47] Peterson, J., and A. Thom. "Group cocycles and the ring of affiliated operators." Preprint available at: http://arxiv.org/abs/0708.4327v1.

[48] Serre, J.-P. Arbres, Amalgames, sl 2 . Paris: Société Mathématique de France, 1977. 
[49] Szőkefalvi-Nagy, B. "On uniformly bounded linear transformations in Hilbert space." Acta University Szegediensis Scientiarum Mathematicarum 11 (1947): 152-7.

[50] Varopoulos, N. T. “On an inequality of von Neumann and an application of the metric theory of tensor products to operators theory." Journal of Functional Analysis 16 (1974): 83-100.

[51] Weyl, H. "Repartición de corriente en una red conductora." Revista de Matemáticas HispanoAmericana 5 (1923): 153-64.

[52] Wilson, D. B. “Generating random spanning trees more quickly than the cover time," 296-303. Proceedings of the 28th Annual ACM Symposium on the Theory of Computing. New York: Association for Computing Machinery, 1996. 